\title{
Manifestaciones cutáneas de la infección por virus de inmunodeficiencia humana en niños de Santiago de Chile
}

\author{
Paula Muñoz M., Orietta Gómez H. y Amaranta Luzoro V.
}

\section{Skin diseases in human immunodeficiency virus positive children from Santiago, Chile}

Children infected with human immunodeficiency virus (HIV) may develop severe, refractary mucocutaneous manifestations that may be the initiating symptom of HIV infection. In this study we examined the skin of all HIV positive children receiving medical care in the public health care system in Santiago, Chile,. We detected mucocutaneous manifestations in $37 / 66$ (56\%) children from 7 months to 12 years of age. The most commonly encountered dermatologic manifestations were of infectious origin, mostly fungal $(7.5 \%)$ and viral $(7.5 \%)$ infections. With the increase in pediatric HIV patients worldwide, it is important to recognize skin manifestations of HIV positive children. This is the first published series of skin diseases in HIV positive children in Chile.

Key words: skin diseases, HIV, children, mucocutaneous manifestations.

Palabras clave: manifestaciones cutáneas, VIH, niños.

\section{Introducción}

$\mathrm{E}$ 1 síndrome de inmunodeficiencia adquirida, SIDA, fue reconocido como una nueva entidad clínica en junio de 1981 en E.U.A. ${ }^{1,2}$. En 1984, se demostró que la enfermedad estaba ligada a un retrovirus linfocitotrópico de las células $\mathrm{T}$ helper, al que se denominó virus de la inmunodeficiencia humana tipo 1. Muy poco después se identificó un segundo virus de la inmunodeficiencia humana, el VIH-2 ${ }^{1,3}$. A diciembre del año 2000, se estimaba que habían fallecido en el mundo desde el inicio de la epidemia 21,8 millones de personas y que 36,1 millones estaban infectados con VIH o padecían el SIDA $^{4}$. Alrededor de 16 millones corresponden a mujeres y 1,4 millones a personas bajo 15 años de edad ${ }^{5}$. La infección por VIH en niños se define como aquella que ocurre antes de los 13 años de edad. En 1982, se informó al Centro de Control y Prevención de Enfermedades (CDC) de E.U.A., el primer caso de SIDA en un niño ${ }^{6}$, desde entonces, el número de niños infectados por el VIH, ha aumentado rápida y sostenidamente siendo una causa importante de enfermedad grave y muerte en niños de todo el mundo ${ }^{7,8}$.
En los niños de la mayor parte del mundo, la transmisión perinatal es la más importante, con valores cercanos al 90\%7. Según estudios prospectivos, el riesgo de que una mujer infectada con VIH transmita el virus al producto de la gestación varía entre 15 y $45 \% \%^{7-10}$.

En Chile, el primer caso de SIDA en un niño fue notificado en $1987^{\circ}$. Desde esa fecha hasta marzo de 2001 se habían diagnosticado 145 niños infectados. De ellos han fallecido 36, con una letalidad de 24,5\%. Desde 1989 hasta marzo de 1994 había una tasa de transmisión vertical de 35,4\%, por lo que en 1995 se inició en Chile la utilización del protocolo ACTG 076 (Pediatric AIDS Clinical Trials Group), según el cual se administra zidovudina a la madre durante las últimas semanas del embarazo y durante el parto y al recién nacido durante las primeras seis semanas de vida

Con esta innovación, en 1998 se obtuvo una disminución de la transmisión a $6,9 \%$, lo que determinó una reducción de la tasa de transmisión vertical global a 23\% entre los años 1995 y 1998.

Desde 1998 se ha continuado utilizando la profilaxis, además de otros protocolos en que se agregan otros antiretrovirales
Hospital Clínico Universidad de Chile

Departamento de Dermatología

Recibido: 20 junio 2007 Aceptado: 15 mayo 2008

Correspondencia a:

Paula Muñoz Moller paulantonia@vtr.net

\section{Nota del Editor}

Las estadísticas actualizadas de infección por VIH/SIDA en niños chilenos pueden ser consultadas en: M. García O. y A. Olea O. Evolución y situación epidemiológica de la infección por virus de inmunodeficiencia humana y síndrome de inmunodeficiencia adquirida en Chile. Rev Chil Infect 2008; 25 (3): 162-70. 
Entre 1998 y 2001, se logró una tasa de transmisión vertical de $3 \%$, en el grupo de madres que se sometieron a protocolos de disminución de la transmisión vertical. Sin embargo, hubo madres infectadas por VIH en las que se desconocía esta situación y no tuvieron acceso a medidas de prevención, por lo que considerando las madres que tuvieron acceso a protocolo y las que no lo tuvieron, la transmisión vertical global fue de $15,3 \%{ }^{10}$.

Los niños con infección por VIH pueden presentar una amplia variedad de enfermedades infecciosas e inflamatorias de la piel. Muchas veces estas enfermedades son dermatosis pediátricas frecuentes. Sin embargo, estos trastornos suelen ser más graves, persistentes y más difíciles de tratar que en niños sanos

A veces, los trastornos de la piel o de las mucosas proporcionan un indicio temprano respecto de la presencia de infección por $\mathrm{VIH}$ en niños ${ }^{7,9}$

En Chile no hay estudios destinados a analizar las manifestaciones cutáneas de la infección por VIH en niños.

\section{Objetivos}

- Identificar las principales manifestaciones mucocutáneas que presentan los pacientes pediátricos infectados por VIH atendidos en el sistema público de salud en Santiago de Chile.

- Conocer la incidencia de manifestaciones cutáneas durante el transcurso de la infección por VIH en niños.

- Determinar la asociación entre las distintas manifestaciones mucocutáneas y el grado de inmunodeficiencia celular.

\section{Pacientes y Método}

Se realizó examen dermatológico y análisis de las fichas clínicas de todos los niños registrados con infección por VIH en el sistema público de salud en Santiago de Chile.

Se estudió a todos los niños con infección por VIH, bajo 16 años de edad, que asistían a control ambulatorio a los hospitales San Juan de Dios, San Borja Arriarán, Roberto del Río, Sótero del Río, Exequiel González Cortés y Luis Calvo Mackenna, en el período comprendido entre abril de 2002 y abril de 2003.

De las fichas clínicas se recogieron los siguientes datos: sexo, edad, recuento de CD4, carga viral, etapa de infección por VIH y antecedente de dermatosis

Mediante examen físico dermatológico, realizado por un observador entrenado, se determinó la presencia de lesiones mucocutáneas.

Los datos obtenidos se ingresaron en una base de datos y el análisis estadístico se hizo mediante el programa SPSS 10 .

\section{Resultados}

Se estudió un total de 72 niños con infección por VIH, bajo 16 años de edad. Se analizó un total de 66 casos que cumplían con el límite de edad bajo 13 años, 35 de sexo femenino (53\%) y 31 de sexo masculino (47\%), con un rango de edad entre 7 meses y 12 años. El promedio de edad fue 6 años.

En $97 \%$ de los casos el mecanismo de transmisión del VIH fue vertical.

Veintiun por ciento de los niños estudiados tenía carga viral indetectable y $59,1 \%$ tenía una carga viral mayor a 10.000 copias de ARN viral $/ \mathrm{ml}$ (Tabla 1 ).

En promedio, el recuento de linfocitos CD4 fue de $620 / \mathrm{mm}^{3}$, con un mínimo de 5 y un máximo de 3302/ $\mathrm{mm}^{3}$. E1 59,1\% de los niños tenía recuento de CD4 entre 200 y $800 / \mathrm{mm}^{3}$

Según la clasificación en categorías inmunológicas, que considera la edad y el recuento de CD4, el 36,4\% de los niños se encontraba sin supresión, 39,4\% con supresión moderada y el $24,2 \%$ con supresión grave (Tabla 2)

Al considerar la clasificación según categorías clínicas e inmunológicas, 63,6\% de los pacientes estudiados se encontraba en etapa SIDA (Tabla 3).

Tabla 1. Carga viral en 66 pacientes pediátricos incluidos en el estudio

\begin{tabular}{lcc|}
$\begin{array}{l}\text { Carga viral } \\
\text { Copias ARN/ml }\end{array}$ & n niños & \% \\
\hline Indetectable $(<L D E)$ & 14 & 21,0 \\
$0-10.000$ & 12 & 18,2 \\
\hline 10.001 y más & 39 & 59,1 \\
Sin dato & 1 & 1,5 \\
\hline Total & 66 & 100 \\
\hline
\end{tabular}

Tabla 2. Clasificación de 66 pacientes pediátricos incluidos en el estudio según categoría inmunológica (CDC 1994)

Categoría inmunológica

Sin supresión

Supresión moderada

Supresión grave

Total n niños

24

26

16

66
$\%$ 36,4 
Tabla 3. Etapa de infección por VIH/SIDA en 66 pacientes pediátricos incluidos en el estudio

\begin{tabular}{|lcc|} 
Etapa & $\mathbf{n}$ niños & $\%$ \\
N2 & 4 & 6,1 \\
N2-3 & 1 & 1,5 \\
A1-2 & 1 & 1,5 \\
A2 & 4 & 6,1 \\
A3 & 1 & 1,5 \\
B1 & 7 & 10,6 \\
B2 & 5 & 7,6 \\
B3 & 3 & 4,5 \\
C1 & 1 & 1,5 \\
C2 & 8 & 12,1 \\
C3 & 29 & 43,9 \\
En etapificación & 1 & 1,5 \\
Sin dato & 1 & 1,5 \\
\hline Total & 66 & 100 \\
\hline
\end{tabular}

Tabla 4. Antecedentes de dermatosis en $\mathbf{6 6}$ niños con infección por VIH. Santiago, Chile

\begin{tabular}{lcc}
$\begin{array}{l}\text { Antecedente de } \\
\text { lesión en la piel }\end{array}$ & n antecedentes & \% \\
\hline Sin antecedente & 46 & 69,7 \\
Candidiasis & 8 & 33,3 \\
\hline Prúrigo & 5 & 20,8 \\
\hline HSV-1 & 5 & 20,8 \\
\hline Tinea capitis & 2 & 8,3 \\
Molusco contagioso & 1 & 4,2 \\
VVZ & 1 & 4,2 \\
Verrugas & 1 & 4,2 \\
Impétigo & 1 & 4,2 \\
Total & $70 *$ & 100
\end{tabular}

* El total corresponde al número de antecedentes encontrados, que en 4 casos fue 2 . El \% de antecedentes se encuentra en relación a los diagnósticos positivos, excepto sin antecedentes que se encuentra en relación al número de niños observados.

\section{Tabla 5. Frecuencia de hallazgos cutáneos en 66} pacientes pediátricos con infección por VIH, Santiago, Chile

\begin{tabular}{|lcc|}
\hline n hallazgos en piel & n casos & \% \\
\hline Uno & 30 & 45 \\
\hline Dos & 5 & 8 \\
\hline Tres & 2 & 3 \\
Total & 37 & 56 \\
Sin hallazgo en piel & 29 & 44 \\
Total & 66 & 100 \\
\hline
\end{tabular}

El $69,7 \%$ de los niños estudiados no tenía antecedente de lesión cutánea consignada y en los niños que tuvieron antecedente, el diagnóstico más frecuente fue candidiasis $(33,3 \%)$ (Tabla 4).

Cincuenta y seis por ciento de los niños examinados presentó, al momento del examen clínico, una o más lesiones cutáneas (Tabla 5), resultando 46 diagnósticos de dermatosis (Anexo 1).

El detalle de los hallazgos cutáneos observados se muestra en la Figura 1 y en la Tabla 6.

Los hallazgos diversos se refieren a xerodermia, queratosis pilar, hiperpigmentación postinflamatoria e hipertricosis, siendo lo más frecuente la xerodermia, ya que se encontró en nueve $(13,6 \%)$ de los niños examina-

\begin{tabular}{|c|c|c|}
\hline Hallazgo en piel & n diagnósticos & $\%$ \\
\hline Negativo & 29 & 43,9 \\
\hline Prúrigo agudo & 7 & 15,2 \\
\hline Infecciones micóticas & 5 & 10,9 \\
\hline Infecciones virales & 5 & 10,9 \\
\hline Infecciones bacterianas & 2 & 4,3 \\
\hline Infestaciones & 3 & 6,5 \\
\hline Dermatitis seborreica & 4 & 8,7 \\
\hline Dermatitis de contacto irritativa & 4 & 8,7 \\
\hline Acné & 1 & 2,2 \\
\hline Distrofia ungueal & 1 & 2,2 \\
\hline Hallazgos diversos & 14 & 30,4 \\
\hline Total & $75^{*}$ & 100 \\
\hline \multicolumn{3}{|c|}{$\begin{array}{l}\text { * El total corresponde al número de diagnósticos realiza- } \\
\text { dos, que en } 7 \text { niños fue más de uno. El \% de los hallaz- } \\
\text { gos se encuentra en relación a los diagnósticos positivos } \\
\text { excepto el hallazgo negativo que se encuentra en relación } \\
\text { al número de niños observados. }\end{array}$} \\
\hline
\end{tabular}

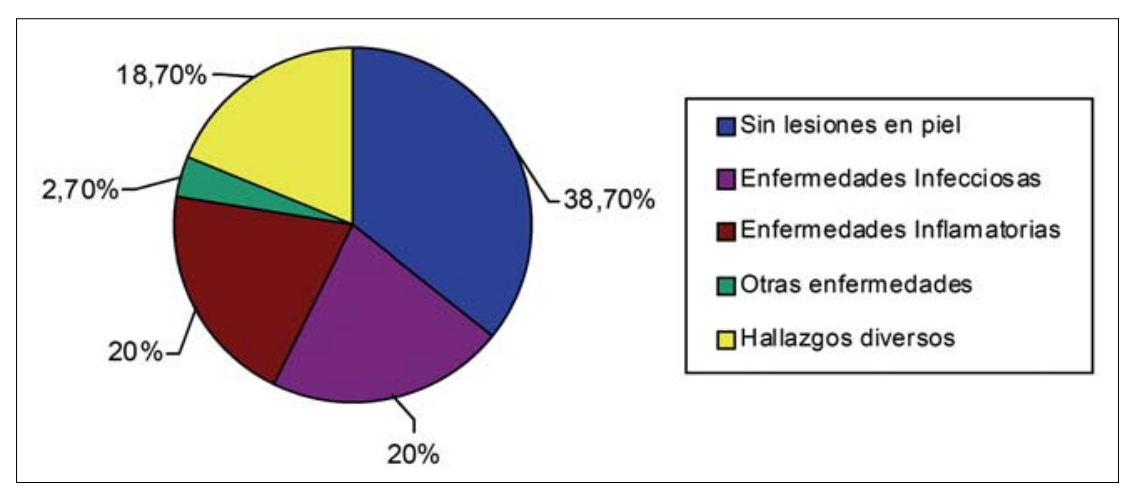

Figura 1. Hallazgos cutáneos en 66 niños con infección por VIH. 
Anexo 1. Fotos clínicas de algunas manifestaciones cutáneas encontradas.

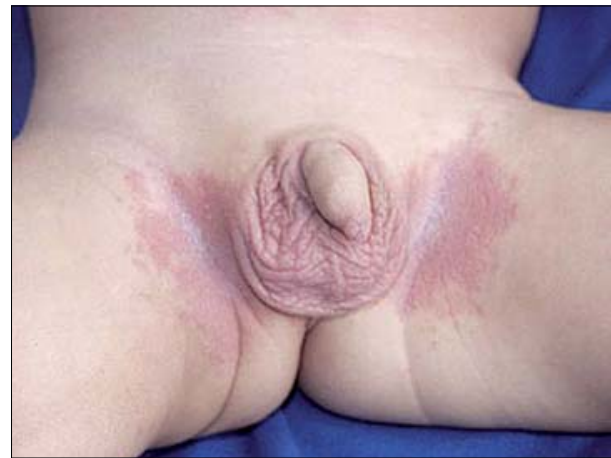

Candidiasis del pañal

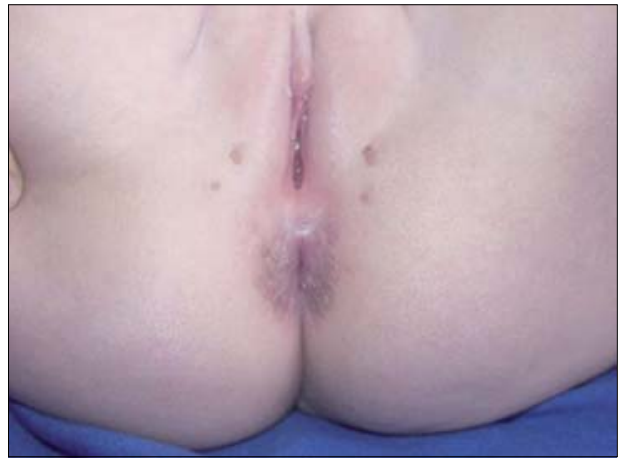

Condilomas acuminados

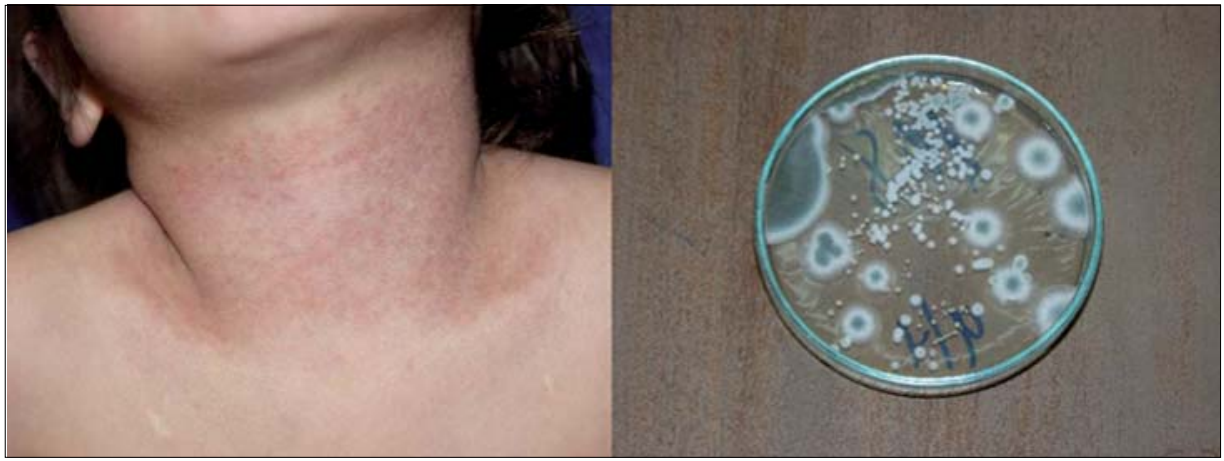

Aspergilosis cutánea

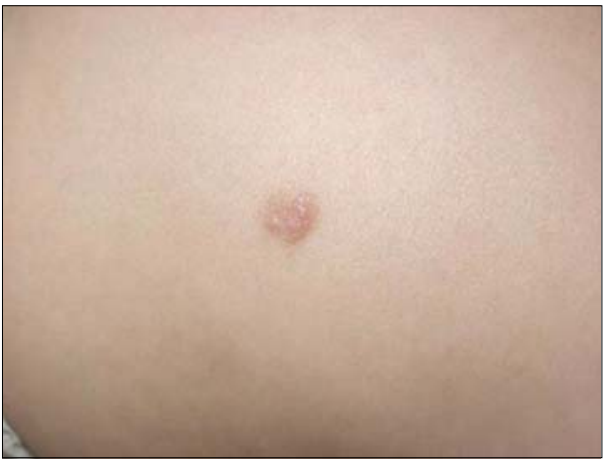

Nódulo escabiótico

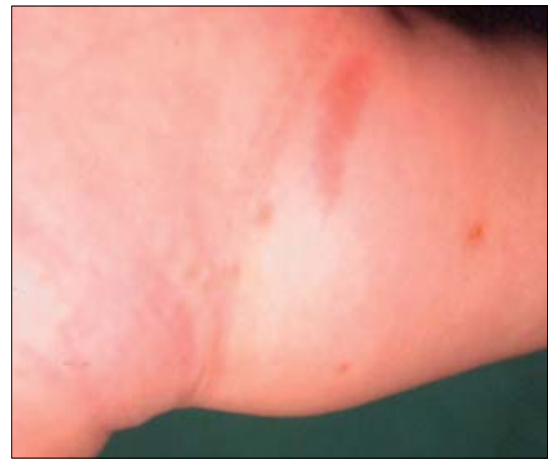

Prúrigo agudo

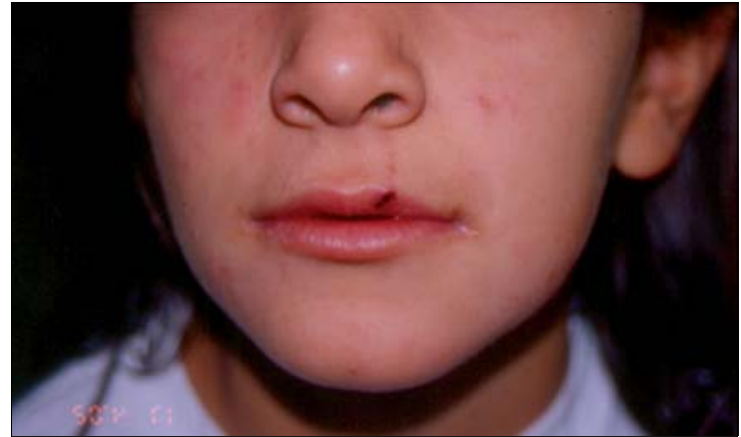

Herpes simplex labial

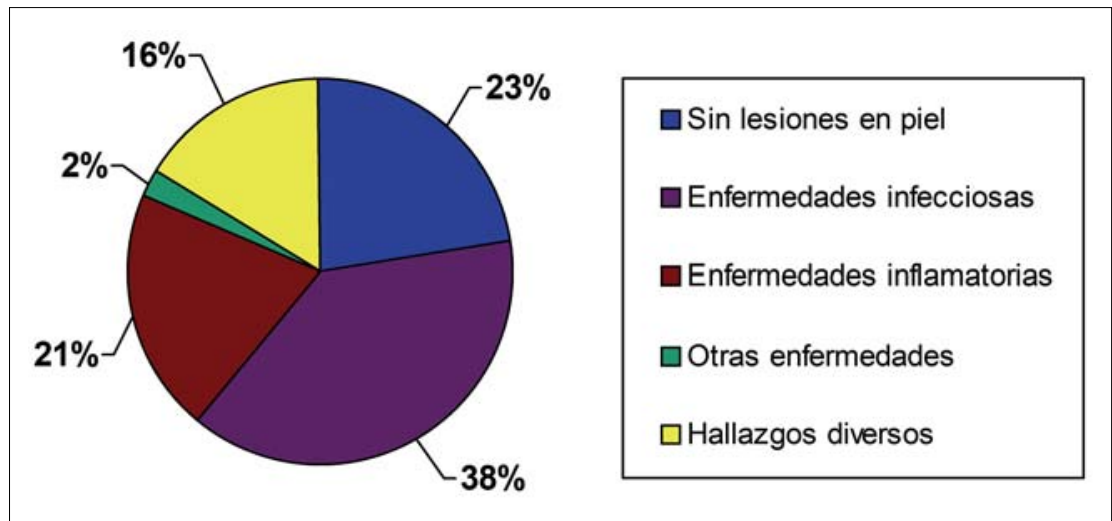

Figura 2. Antecedentes de lesión en piel y hallazgo cutáneo en los niños estudiados.
Tabla 7. Antecedentes de lesión en piel y hallazgos cutáneos en 66 pacientes con infección por VIH, Santiago, Chile

\begin{tabular}{lrc} 
Hallazgo en piel & n diagnósticos & \% \\
Negativo & 21 & 31,8 \\
Infecciones micóticas & 16 & 22,5 \\
\hline Infecciones virales & 13 & 18,3 \\
Infecciones bacterianas & 3 & 4,2 \\
\hline Infestaciones & 3 & 4,2 \\
Dermatitis seborreica & 4 & 65,6 \\
\hline Dermatitis de contacto irritativa & 4 & 55,6 \\
Prúrigo agudo & 11 & 15,5 \\
\hline Acné & 1 & 1,4 \\
Distrofia ungueal & 1 & 1,4 \\
Hallazgos diversos & 15 & 21,1 \\
\hline Total & $92 *$ & 100
\end{tabular}

*El total corresponde al número de diagnósticos realizados, que en 18 niños fue más de uno. El \% de los hallazgos se encuentra en relación al total de diagnósticos positivos, excepto el hallazgo negativo que se encuentra en relación al número de niños observados. 
dos. Cuatro pacientes presentaron dermatitis seborreica, de los cuales tres pacientes tenían 4 años y un paciente 8 años.

Al tomar en cuenta el antecedente de lesión cutánea y el hallazgo en piel al momento del examen clínico, 45 niños $(68,2 \%)$ tuvieron en el transcurso de su enfermedad alguna manifestación cutánea y 10 de ellos $(22,2 \%)$ tuvieron candidiasis. El detalle se observa en la Figura 2 y en la Tabla 7 .

Al analizar la asociación entre grado de inmunosupresión y presencia de dermatosis, se comprobó que los niños con algún grado de supresión inmunológica (moderada o grave) tuvieron un riesgo 3,6 veces mayor $(\mathrm{IC}=1,3-10)$ de presentar alguna manifestación cutánea, en relación a los niños sin supresión $(\mathrm{p}<0,05)$ (Figura 3).

De la misma manera, los niños en etapa SIDA tuvieron un riesgo 6,3 veces mayor de tener alguna dermatosis en relación a los que no estaban en esta etapa $(\mathrm{p}=0,0004)$ (Figura 4).

\section{Discusión}

Los niños con infección por VIH/SIDA atendidos en el sistema público de Santiago de Chile, presentan diversas manifestaciones cutáneas, de las cuales las más frecuentes son las infecciones. Las infecciones micóticas, seguidas de las virales se encontraron en un mayor número de pacientes. La dermatitis seborreica, el prúrigo agudo y la xerodermia fueron otros hallazgos comunes en estos niños

Estos resultados son concordantes con los obtenidos en un estudio realizado en Nueva York, en que se examinó a 21 niños con infección por VIH, de los cuales 13 presentaron manifestaciones dermatológicas, siendo más frecuentes las enfermedades infecciosas y dentro de ellas las infecciones micóticas (candidiasis), seguidas de las infecciones virales ${ }^{11}$.

Algunos estudios latinoamericanos demuestran también que la candidiasis es la manifestación mucocutánea más frecuente en los niños con infección por VIH/SIDA. En Ciudad de México se revisaron las fichas clínicas de 149 pacientes pediátricos infectados con VIH en un período de 10 años, resultando 251 diagnósticos de dermatosis, de los cuales el diagnóstico más frecuente fue la candidiasis oral, en $49 \%{ }^{12}$. También, en República Dominicana y Cuba los niños con SIDA presentaron como lesión dérmica más frecuente la candidiasis ${ }^{13,14}$.

En nuestro estudio se observó que en el transcurso de la infección por $\mathrm{VIH}, 68,2 \%$ de los niños presentó en algún momento alguna manifestación cutánea $\mathrm{y}$ que $22,2 \%$ tuvo candidiasis.

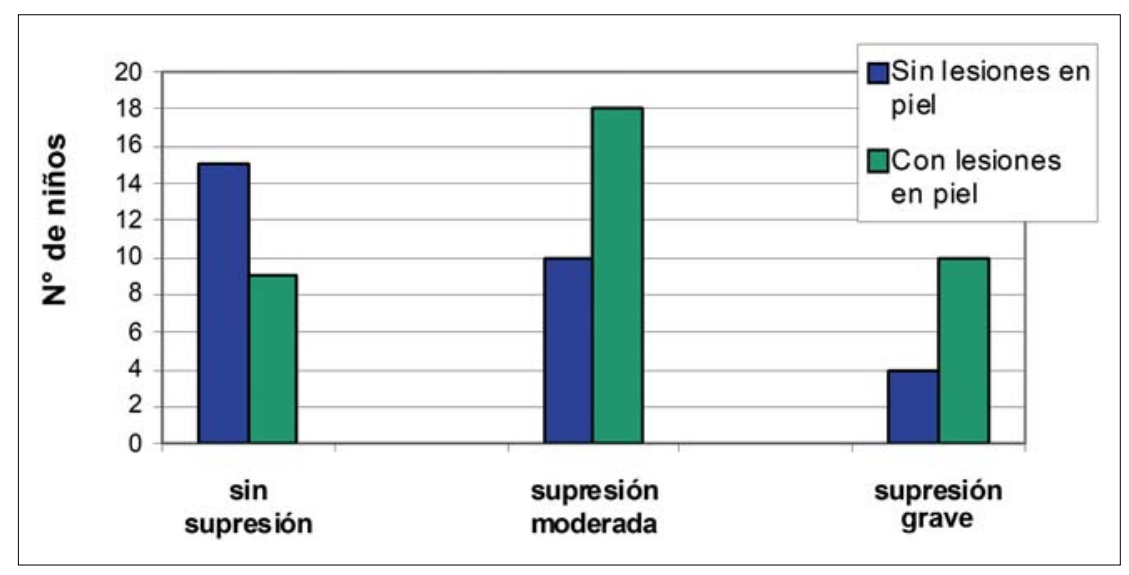

Figura 3. Presencia de dermatosis según estado inmunológico.

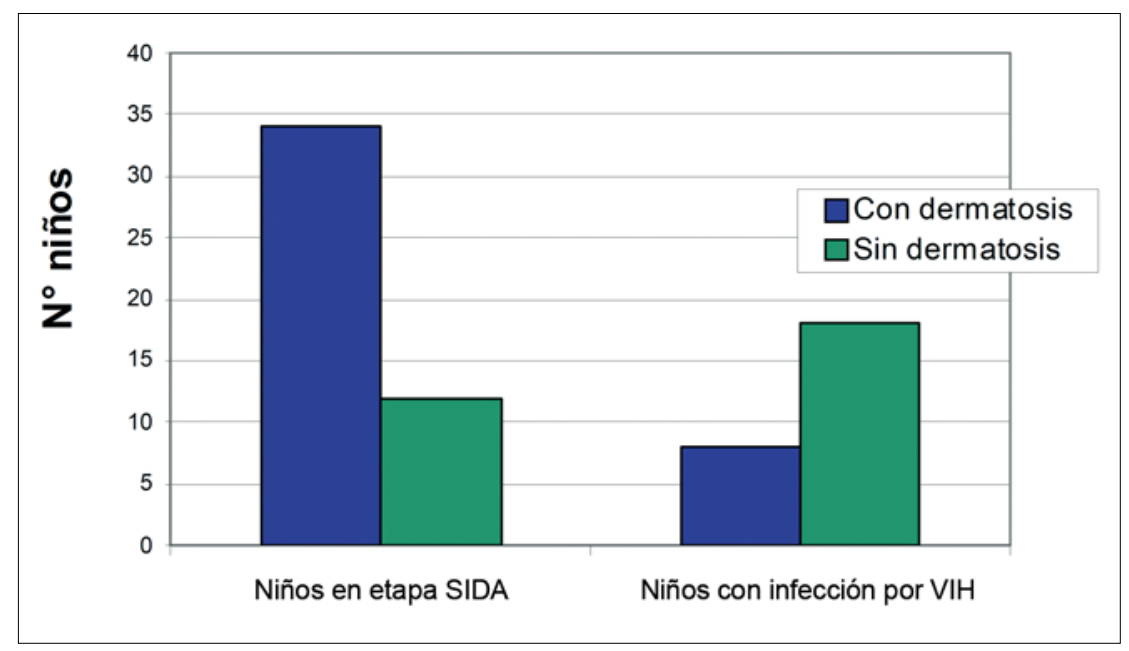

Figura 4. Dermatosis en 66 niños con infección por VIH/SIDA.

Por otra parte, los niños estudiados que tienen algún grado de inmunosupresión celular presentan más manifestaciones cutáneas que los niños que tienen un recuento de linfocitos CD4 normal para su edad. Esto es comparable a lo obtenido en Nueva York, en que la mayoría de los trastornos cutáneos ocurrieron cuando el recuento de células CD4(+) fue menor a 200 células $/ \mathrm{mm}^{3}{ }^{11}$, y con otro estudio realizado en Tailandia, en que se determinó que la prevalencia de los hallazgos mucocutáneos en niños con infección por VIH aumenta en tanto que el porcentaje de células CD4 disminuye ${ }^{15}$. A su vez, los niños examinados que están en etapa de SIDA tienen más riesgo de presentar alguna lesión en piel.

En Chile, la mayoría de los niños en que el diagnós- 
tico de infección por VIH se plantea por patología, están ya en etapa de SIDA y, a pesar de los excelentes resultados de las medidas de reducción de la transmisión vertical del VIH, el número de niños infectados continúa en aumento ${ }^{10}$. Esto es porque en la mayoría de los niños en los que se diagnóstica infección por VIH no se tiene el antecedente de infección en la madre, lo que representa un desafío para los pediatras y obliga a estar atentos a las manifestaciones precoces de esta infección para un diagnóstico y tratamiento oportunos

La importancia de este trabajo radica en conocer las principales dermatosis asociadas a la infección por VIH en los niños de nuestro país, ya que pueden ser una de las primeras manifestaciones de la enfermedad fácilmente observables.

Por las características del tipo de estudio realizado, transversal y descriptivo, no es posible determinar un potencial marcador cutáneo temprano de infección por VIH/SIDA. Además, los hallazgos de piel observados pueden presentarse también en población pediátrica sana. La diferencia estaría dada en que estas dermatosis comunes suelen ser más graves, de presentación atípica, con tendencia a recurrir y con menor respuesta al tratamiento en los niños infectados con el VIH.

Para determinar si estas enfermedades de piel son más frecuentes en niños con infección por VIH/SIDA, futuros estudios podrían comparar los resultados con un grupo control de niños sanos
Agradecimientos. A los niños y sus familias, al personal del hogar Santa Clara y a los médicos y personal de los hospitales San Juan de Dios, Félix Bulnes, San Borja Arriarán, Roberto del Río, Sótero del Río, Exequiel González Cortés y Luis Calvo Mackenna.

\section{Resumen}

Los niños infectados por el virus de la inmunodeficiencia humana, VIH, pueden presentar variadas manifestaciones mucocutáneas, muchas veces más graves y más difíciles de tratar que las de los niños sanos. Los trastornos de la piel o de las mucosas pueden proporcionar un indicador temprano de infección pediátrica por VIH. Para este estudio fueron examinados todos los niños infectados con VIH, atendidos en el sistema público de salud en Santiago de Chile. Registramos 66 niños con rango de edades entre 7 meses y 12 años. Las manifestaciones muco-cutáneas fueron encontradas en 37 (56\%). Los hallazgos dermatológicos más observados fueron las enfermedades infecciosas, de las cuales las infecciones micóticas y virales fueron las más frecuentes. Con el aumento del número de pacientes pediátricos infectados por el VIH en el mundo, es muy importante reconocer las manifestaciones cutáneas, ya que pueden permitir realizar un diagnóstico precoz de la infección por VIH. Este es la primera serie clínica publicada acerca de las enfermedades de la piel en niños con infección por VIH en Chile.

\section{Referencias}

1.- Sepúlveda C. Historia y orígenes del SIDA. Sepúlveda C, Afani A, editores. SIDA, Tercera Edición. Chile. Editorial Mediterráneo; 2002, p. 1-9

2.- Pérez C. Historia natural de la infección por VIH. Sepúlveda C, Afani A, editores. SIDA. Tercera Edición. Chile. Editorial Mediterráneo; 2002, p. 29-38.

3.- Vial P. El virus de la inmunodeficiencia humana. Sepúlveda C, Afani A, editores. SIDA. Tercera Edición. Chile. Editorial Mediterráneo; 2002, p. 63-95

4.- Arredondo A. La epidemia del VIH/SIDA en Chile y el mundo. Sepúlveda C, Afani A, editores. SIDA. Tercera Edición. Chile. Editorial Mediterráneo; 2002 p. $10-28$

5.- Comisión Nacional de SIDA. Situación epidemiológica de la infección por virus de la inmunodeficiencia humana/síndrome de inmunodeficiencia adquirida en Chile.
Diciembre 2000. Rev Chil Infect 2001; 18: 261-70.

6.- Centers for Disease Control: Unexplained immunodeficiency and opportunistic infection in infants. New York, New Yersey and California. MMWR Morb Mortal Wkly Rep 1982; 31: 665-7.

7.- Prose N. Mucocutaneous disease in pediatric human immunodeficiency virus infection. Pediatr Clin North Am 1991; 38: 977-90.

8.- Wu E. Infección por VIH en niños. Sepúlveda C, Afani A, editores. SIDA. Tercera Edición. Chile. Editorial Mediterráneo; 2002, p. 457-87.

9.- Prose N. Cutaneous manifestations of HIV infection. Harper J, Oranje A, Prose N. Textbook of Pediatric Dermatology. Blackwell Science 2000; 1: 362-7

10.- Comité Nacional de SIDA Pediátrico. Situación actual de la infección por virus de inmunodeficiencia humana en niños. Rev Chil Infect 2001; 18: 271-4. 11.- Lim W, Sadick N, Gupta A, Kaplan M,
Pahwa S. Skin diseases in children with HIV infection and their association with degree of immuno-suppression. Int J Dermatol 1990; 29: 24-30.

12.- Soriano-Hernández Y L, Tamayo-Sánchez L, Xochihua-Díaz L, Durán-McKister C, Orozco-Covarrubias L, Ruiz-Maldonado R. Manifestaciones cutáneas en 149 pacientes pediátricos infectados con VIH-SIDA. Bol Méd Hosp Infant Méx 2001; 58: 163-7.

13. - Suriel D, García Y, Soto S, Rodriguez J. Lesiones dérmicas en niños seropositivos al virus de inmunodeficiencia humana. (VIH) Acta Méd Domin 1997; 19: 189-92.

14. - Santana J A, Hernández B, Díaz de la Rocha A, Martínez I, Negrín L. Manifestaciones dermatológicas en pacientes con SIDA. Rev Cubana Med Gen Integr 2000; 16: 287-90.

15.- Wananukul S, Deekajorndech T, Panchareon C, Thisyakorn U. Mucocutaneous findings in pediatric AIDS related to degree of immunosuppression Pediatr Dermatol 2003; 20: 289-94 